\title{
Comparison of Nonstress Test and Light stimulation Test and their correlation with fetal outcome
}

\author{
Khanam D ${ }^{1}$, Bano $I^{2}$ \\ ${ }^{1}$ Dr. Deeba Khanam, Senior Resident, Department of Obstetrics and Gynaecology, 2Prof Imam Bano, Professor, \\ Department of Obstetrics and Gynaecology. Both are affiliated with J.N Medical College A.M.U Aligarh, U.P, India
}

Address for Correspondence: Dr. Deeba Khanam, Emai, khanamdeeba@gmail.com

\begin{abstract}
Introduction: Non Stress Test serves an important function in assessment of fetal wellbeing, but false positive results limit its role. Light stimulation stimulates the fetus, speeds up the test and catalyses its rate limiting step. This study has compared simple Non Stress Test and Light stimulation test and there correlation with various fetal outcomes. This study helps us to assess whether light stimulation be used as a better alternative or an adjunct to traditional Nonstress test. Material and Methods: A total of 220 patients above 34 weeks of pregnancy were taken. 110 pts underwent 20 minutes of Nonstress test and 110 underwent Halogen light stimulation which was given transabdominally for 10 seconds and it was repeated every 10 minutes for a maximum up to three times. This allocation was done randomly. Non reactive Nonstress test were then stimulated with Halogen light. Results: The test showed that sensitivity of Nonstress test is greater than Light stimulation test, but specificity, positive predictive value, and negative predictive value is lesser than Light stimulation test .Out of 36 Non reactive NSTs after stimulation with light 18 became reactive. Conclusion Nonstress test is considered as a screening test and Light stimulation test can be used as an adjunct to Nonstress test as it decreases the number of false positive results and increases the specificity of the test. Clinical outcomes are more closely related with LST, but more studies are required to arrive at the final conclusions.
\end{abstract}

Keywords: Non-Stress Test, Light Stimulation Test, Positive Predictive Value, Negative Predictive Value.

\section{Introduction}

Assessment of fetal wellbeing is of prime importance in modern day obstetrics. Various tools have been investigated since the time of Francois Mayor, 1818 who first heard the sound of fetal heart when he placed ear on the maternal abdomen to the present era of electronic fetal monitoring [1].

Fetal heart rate monitoring is considered to be reliable for fetal assessment and from time to time amendments are made in the methods of assessment.

A rapid and predictable antenatal screening test is of great value in assessment of fetus at risk, thus Nonstress test evolved and was accepted as primary fetal surveillance tool. NST is popular due to ease of its application and modest technical requirement. The point not in favour of it is high number of false positive cases mainly due to foetuses which are asleep or are hypoactive [2]. These foetuses could be made active by

Manuscript received: $1^{\text {st }}$ Dec 2014

Reviewed: $6^{\text {th }}$ Dec Aug 2014

Author Corrected: $19^{\text {th }}$ Dec 2014

Accepted for Publication: $22^{\text {th }}$ Dec 2014

International Journal of Medical Research and Review stimulating them by number of means like manual stimulation, glucose ingestion, vibroacoustic stimulation and light stimulation. Light stimulation is the present field of interest. Various light sources were tested and it was concluded that halogen bulb was safe, penetrated effectively and was the light source of choice for fetal stimulation [3]. First fetal movement in response to light was reported by Polishak et al in 1975[4]. Peleg G, Goldman JA et al in 1980 [5] also studied effect of light stimulation on fetus. Tatsumura et al [6] in 1991 reported an increase in response to light proportional to gestational age. Tuangsit Watagnara et al [7] in 2006 studied the shortening of NST using light stimulation.

These studies concluded that light stimulation does provoke the fetus and response is obtained in the form of fetal heart rate stimulation, reduces the number of false positive cases[8], but results of shortening of testing time are variable. In my present study that has been carried out at JNMCH AMU, Aligarh, we have tried to compare Nonstress test and Halogen Light stimulation test by following the fetal outcome and

Available online at: $\underline{\text { www.ijmrr.in }} 35$ | P a g e 
Research Article

recording the number of foetuses who had meconium, fetal distress, apgar scores less than 7, type of delivery they underwent and foetuses who died .

The degree to which the results of NST and LST correlated with the fetal outcome gave an idea regarding the sensitivity, specificity, negative and positive predictive value of the test and thus helps in choosing which to be used as more reliable screening test.

\section{Materials and Methods}

The present study has been carried out in department of obstetrics and Gynaecology, Jawaharlal Nehru Medical College AMU, Aligarh in patients attending outpatient department, antenatal clinic and indoor department of Obstetrics and gynaecology. 220 patients above 34 weeks of gestation were selected for the study.110 were randomly selected in each group undergoing Nonstress test and Light stimulation test.

\section{Equipments}

The equipment used was an Avalon FM-20 monitor which gave continuous recordings of fetal heart rate on a thermal graph paper. The strip chart provided permanent record of FHR pattern. Halogen light source consists of a halogen bulb of 35 watt light intensity which is equal to 1 lakh candle power strength, covered by a rubber encasement which is a non conductor of heat.

An ammeter and voltmeter were also connected in circuit to measure the power provided to halogen lamp and regulate the intensity of light. This was assembled and prepared in the polytechnic lab of AMU, Aligarh.

The light stimulation test was conducted in the following manner. Halogen light source was placed at patient's abdomen on the fetal head after confirming the position of fetal head.

Light stimulation of power 35 watt was given to the patient for 10 seconds and response to stimulation was noted on CTG paper in the form of acceleration. If no acceleration was noted then stimulus was repeated after 10 minute interval, for maximum up to 3 times before declaring the result to be nonreactive.

Fetal outcome was recorded in the form meconium, fetal distress, apgar scores less than 7, NICU admissions, mode of delivery and mortality.

\section{Results}

The present study consists of 220 patients which includes both high risk and low risk group and they underwent Non Stress Test and Light stimulation test randomly and each group had 110 patients.

\section{Interpretation of CTG}

Out of 110 patients $74(67 \%)$ had reactive traces and $36(33 \%)$ had nonreactive traces. Out of 110 patients undergoing LST 92 were reactive and 18 were nonreactive which makes $83.6 \%$ and $16.3 \%$ respectively.

\section{Correlation of NST with Meconium}

Out of 110 patients 28 had meconium in liquor. Among 74 reactive 16 had meconium that accounts to $21.6 \%$ and out of 36 nonreactive 12 had meconium which makes $33.33 \%$. This result shows that occurrence of meconium is higher in patients with nonreactive NST.

\section{Correlation of NST with Fetal Distress}

This study shows that out of 36 non reactive cases 17 had fetal distress that makes $47.22 \%$. In 74 reactive cases fetal distress was present in 17 cases which make $22.9 \%$. This result shows that incidence of fetal distress is more common in non reactive cases

\section{Correlation of NST with Low Apgar score}

In the present study it has been seen that total number of patients who had low apgar scores that is $<7 / \Upsilon 10$ and $8 / 10$ were 26 . Out of 74 reactive cases number of low apgar scores were $14(18.9 \%)$ and out of 36 nonreactive cases number of low Apgar score were 12 (33.33\%). This result showed that the incidence of low Apgar score was higher in nonreactive cases.

\section{Correlation of NST with NICU Admission}

Among 36 nonreactive cases there were 10 NICU admissions that accounts to $27.7 \%$. Out of 74 reactive cases there were 12 NICU admissions that make $16.2 \%$. This result shows that incidence of NICU admissions is more common in group with non reactive non stress test.

\section{Correlation of NST with Mode of Delivery}

The above result shows the relationship between numbers of caesareans and normal deliveries with the NST. Among 36 non reactive cases 20 were caesareans which accounts to $55.5 \%$ and out of that 15 were for fetal distress which makes $41.6 \%$. Out of 74 reactive cases 15 were caesareans and 59 were normal deliveries therefore percentage of caesareans is $20 \%$ and out of 15 caesareans 10 were for fetal distress and that makes 
Research Article

$13.5 \%$. So the result suggests that the number of caesareans as well as number of caesareans for fetal distress were more in nonreactive cases.

\section{Correlation of NST with Mortality}

Out of 74 reactive cases there was no mortality and out of 36 nonreactive cases there were 3 mortalities. This result shows that mortality was more common in patients who had non reactive NST.

\section{Light Stimulation Test}

Out of 110 patients undergoing light stimulation test 92 were reactive and 18 were nonreactive that is equal to $83.6 \%$ and $16.3 \%$ respectively.

\section{Correlation of Light Stimulation with Meconium}

In the present study 110 patients were considered for light stimulation test, out of them 92 were reactive and 18 were not reactive. 10 patients out of 18 non Reactive cases were found to be having meconium that makes $55 \%$ and 19 Patients out of 92 reactive cases were found to be having meconium which accounts to $20.6 \%$. This result shows that incidence of meconium staining is more in non reactive cases.

\section{Correlation of LST with Fetal Distress}

Out of 18 nonreactive cases 10 patients had fetal distress and out of 92 reactive cases 24 had fetal distress.55.5\% of nonreactive cases had fetal distress and $26.08 \%$ of reactive cases had fetal distress as shown below. This result shows that incidence of fetal distress was more common in nonreactive cases.

\section{Correlation of LST with Apgar score}

This result shows that out of 18 non reactive cases 10 patients had Apgar score $<7 / 10$ 8/10, which makes $55.5 \%$. of total 92 reactive cases 16 had apgar scores
$<7 / 10$ and $8 / 10$ which is equal to $17.3 \%$. This result shows that incidence of low apgar scores is more common in non reactive cases.

\section{Correlation of LST with NICU Admissions}

Out of 18 non reactive cases 10 babies were admitted to NICU that makes $55.5 \%$. Out of 92 reactive cases 12 were admitted to NICU which is equal to $13.4 \%$ as shown below in table 13 and bar Chart 13. This result shows that incidence of NICU admission is more in nonreactive cases.

\section{Correlation of LST with Mode of Delivery}

Out of 18 nonreactive cases 7 patients underwent caesareans and 11 had normal deliveries that are $38.8 \%$ had caesareans and $61.1 \%$ had normal deliveries. Out of 7 caesareans 5 were for fetal distress which makes 27.7\% Among 92 reactive cases 32 had caesareans and 60 had normal deliveries that is $34.7 \%$ had caesareans and $65.2 \%$ had normal deliveries. Out of 32 caesareans 20 were for fetal distress that makes $21.7 \%$. This result shows that caesarean section rate for fetal distress is more in patients with nonreactive cases.

\section{Correlation with Mortality}

Out of 18 nonreactive cases there were 3 mortalities that make $16.6 \%$ and out of 92 cases there were no mortalities. This result shows that incidence of mortality is more in non reactive cases.

This is how comparison is done between a simple Nonstress test and Light stimulation test by studying various fetal outcomes and thus helps us to choose which test we prefer in our clinical scenario so as to get accurate and quick results. The table given below shows the comparison of two tests with respect to each variable.

Table 1:- Comparison of NST and LST

\begin{tabular}{|l|l|l|l|l|l|l|l|l|}
\hline \multirow{2}{*}{} & \multicolumn{2}{|l|}{ Sensitivity } & \multicolumn{2}{l|}{ Specificity } & \multicolumn{2}{l|}{ Positive predictive value } & \multicolumn{2}{l|}{ Negative predictive value } \\
\cline { 2 - 10 } & NST & LST & NST & LST & NST & LST & NST & LST \\
\hline Meconium & $42.8 \%$ & $35.7 \%$ & $70.7 \%$ & $90.5 \%$ & $33.3 \%$ & $55.5 \%$ & $78.3 \%$ & $80.4 \%$ \\
\hline FD & $50 \%$ & $29.4 \%$ & $75 \%$ & $89.4 \%$ & $47.2 \%$ & $55.5 \%$ & $77.2 \%$ & $73.9 \%$ \\
\hline $\begin{array}{l}\text { Apgar score } \\
<7 / 10\end{array}$ & $53.8 \%$ & $38.4 \%$ & $71.4 \%$ & $90.4 \%$ & $18.9 \%$ & $55.5 \%$ & $66.6 \%$ & $81.8 \%$ \\
\hline $\begin{array}{l}\text { NICU } \\
\text { Adm. }\end{array}$ & $45.45 \%$ & $45.45 \%$ & $70.5 \%$ & $90.9 \%$ & $27.7 \%$ & $55.5 \%$ & $70.5 \%$ & $86.9 \%$ \\
\hline Mode of Delivery & $57.4 \%$ & $17.9 \%$ & $78.5 \%$ & $84.5 \%$ & $55.5 \%$ & $38.8 \%$ & $79.3 \%$ & $65.2 \%$ \\
\hline Expiry & $100 \%$ & $100 \%$ & $69.5 \%$ & $85.9 \%$ & $8.3 \%$ & $16.6 \%$ & $100 \%$ & $100 \%$ \\
\hline
\end{tabular}

Reducing Number of Non Reactive Cases 
Research Article

Out of 36 nonreactive cases 18 became reactive after light stimulation and 18 stayed nonreactive. Among 18 reactive cases 15 had good fetal outcome and of 18 nonreactive cases 14 had bad clinical outcomes in the form of low apgar scores.

\section{Discussion}

Ante partum fetal wellbeing assessment is the mainstay of modern day obstetrics and key to timely manipulations and successful outcome. Various methods have been proposed for fetal wellbeing assessment. The ideal method should be easy performing, inexpensive, with quickly available results and have a higher degree of correlation with fetal outcome

NST is based on the principle that a normal healthy fetus with intact and well oxygenated autonomic nervous system reacts to spontaneous fetal movements with fetal heart rate acceleration, if not then we can predict that there is something wrong with the fetus but not necessarily as poor outcomes like meconium staining, fetal distress, low apgar scores, NICU admissions and mortality are also associated with reactive foetuses as shown in our study, in other ways this test is less specific. The result of this study shows that poor outcomes are more associated with nonreactive NST. Schiffrin et al, [9] stated that non reactive NSTs are more associated with poor fetal outcomes. This test has the advantage that it has very low false negative rates so it is very unlikely for it to miss a compromised fetus which is very important in considering a test as a screening test and there are no contraindications to the test, it is very easy, inexpensive and the basis of reactive and nonreactive is standard and doesn't vary all over the world. The major drawback is that it has high number of false positive cases due to sleeping or hypoactive foetuses [2].

This results in unnecessary interventions and increased incidence of operative deliveries. To overcome these difficulties, stimulation of fetus with various means was experimented and light stimulation being the present area of interest.

LST and its correlation with various fetal outcomes have been evaluated in this study. As been stated earlier halogen light is considered for stimulating the fetus. This test basically is based on the fact that light exposure results in stimulation of the fetus, makes it move producing fetal heart rate acceleration as studied for the first time by Polishak et al [4]. The foetuses which are asleep and hypoactive get stimulated and respond to the light exposure thereby reducing the chances of the test being termed nonreactive and so makes it more specific and decreases the number of false positive cases [10]. It also reduces the testing time as stated by Bruno $\mathrm{j}$ Cardi et al [8]. The clinical outcomes are more closely related to results of LST as its PSV, NPV and specificity is more than NST as found in our study. The drawback being this halogen light apparatus has to be prepared in addition to Avalon FM monitor which is required in NST and LST both, with all the required prerequisites that is halogen light source, voltmeter, ammeter etc so increases the cost. Patient becomes anxious and curious when halogen bulb case is placed on their abdomen and so consent and counselling is required in this case but overall there are no contraindications and the safety of halogen light has been studied well [3].

\section{Conclusion}

From the present study it can be concluded that LST results in fetal heart rate acceleration by propelling the fetus to move by means of illumination. This results in decreasing the number of non reactive NST cases and hence unnecessasary interventions in the form of labour induction, caesarean sections are reduced. The need for tests like colour Doppler and biophysical profile is also reduced and labour process is made more predictable.

The study shows that NST is more sensitive and is a dependable test for ante partum fetal surveillance but we should not rely on it as a sole screening test as it has high false positive rates. On other hand LST cannot be routinely used in all the cases as it is less sensitive and increases the patient's anxiety and apprehension.LST when combined with NST especially in nonreactive cases, improves the predictability of fetal outcome and allows the prenatal resources to be used more efficiently.

Thus it can be concluded that halogen light stimulation test is an effort in modern obstetrics to assess fetal wellbeing and can be used as an adjunct to traditional NST especially the non reactive ones, and can also be used in patients with impaired hearing where vibroacoustic stimulation is not feasible. Large scale studies are required to further establish the efficacy of the test.

Funding: Nil

Permission from IRB: Yes

Conflicts of interest: The authors report no conflicts of interest 
Research Article

\section{References}

1. Mayor, F. (1818). Bruits du coeur du foetus. Biblioth Universelle des Sciences et Arts, 9, 248

2. Imam Bano, Nasreen Noor .Intrapartum. vibroacoustic stimulation test and cardiotocography for the Prediction of Neonatal outcom.Journal of SAFOG. 2012;Sep-Dec:4(3):141-143. Available at http://www.jaypeejournals.com/eJournals/ShowText.asp $\mathrm{x} ? \mathrm{ID}=4193 \&$ Type $=$ FREE $\&$ TYP $=$ TOP $\& I N=$ eJournals/ images $/$ PPLGO.gif $\&$ IID $=328 \&$ isPDF $=$ YES

3. Brittany B. Rayburn, Daniel P. Theele, D.V.M, Jay M. Bolnick, William F. Rayburn. Selecting an external light source for fetal biophysical testing. J Reprod Med 2004;49:563-565.

4. Polishak W, Laufer N, Sadvosky E.Fetal reaction to external light. Isr. Medicine 1975; vol 89:395-396

5. Dan Peleg, Jack A.Goldman.Fetal heart rate acceleration in response to light Stimulation äs a clinical measure of fetal well-being. A preliminary report. J Perinatal Medicine. 1980; vol 8: 38-41
6. Tatsumura Studies on features of fetal movement and development of human fetus with use of feta actogram Nihon Sanka Fujinka Gakkai Zassh1991;43[8]:864-873

7. Isarin Thanaboonyawat, Tungsit Watangnara, Dittakarn Boriboonhiransarn, Pornpen Somnaiviboonchart BN, Tontisirin BN. Effect of halogen light in fetal stimulation for fetal well-being assessment. J Med Assoc Thai 2006; 89:1376-1380.

8. Bruno j Cardi et al. Effect of halogen light stimulation in non stress testig.,American journal of Obstetrics and Gynecology 2004;vol 190(5):1470-1472. http://dx.doi.org/10.1016/j.ajog.2004.02.031.

9. Schiffrin BS, Foye G, Amato J, Kates R, Mackenna J. Routine fetal heart rate monitoring in the antepartum period. Journal obstetrics and gynecology. 1979;54:2125.

10. Fatemeh Rahimikian, Tahereh Rahiminia, Maryam Modarres, Abbas Mehran. Effect of halogen light stimulation on nonreactive pattern of nst . Iran J Nurs Midwifery Res. 2013 Mar-Apr; 18(2): 112-116.

\section{How to cite this article?}

Khanam D, Bano I. Comparison of Nonstress Test and Light stimulation Test and their correlation with fetal outcome. Int $J$ Med Res Rev 2015;3(1):35-39. doi:10.17511/ijmrr.2015.i1.07 